Discrete Comput Geom 30:343-353 (2003)

DOI: $10.1007 / \mathrm{s} 00454-003-0015-6$

Geometry

\title{
A Volume Formula for Medial Sections of Simplices
}

\author{
István Talata* \\ Department of Mathematics, The University of Michigan, \\ East Hall, 525 East University Avenue, Ann Arbor, MI 48109-1109, USA \\ talata@math.lsa.umich.edu
}

\begin{abstract}
Let $S_{d}$ be a $d$-dimensional simplex in $\mathbb{R}^{d}$, and let $H$ be an affine hyperplane of $\mathbb{R}^{d}$. We say that $H$ is a medial hyperplane of $S_{d}$ if the distance between $H$ and any vertex of $S_{d}$ is the same constant. The intersection of $S_{d}$ and a medial hyperplane is called a medial section of $S_{d}$. In this paper we give a simple formula for the $(d-1)$-volume of any medial section of $S_{d}$ in terms of the lengths of the edges of $S_{d}$. This extends the result of Yetter [5] from the three-dimensional case to arbitrary dimension. We also show that a generalization of the obtained formula measures the volume of the intersection of some analogously chosen "medial" affine subspace of $\mathbb{R}^{d}$ and the simplex.
\end{abstract}

\section{Introduction}

In a recent paper by Yetter [5] a simple formula is given for the area of a parallelogram whose vertices are the midpoints of four edges of a tetrahedron. There the area is expressed in terms of the lengths of the edges of the tetrahedron. Note that the parallelogram considered there can be always obtained as the intersection of the tetrahedron and a plane parallel to two opposite edges of the tetrahedron and lying at equal distance from them.

In this paper we consider a natural generalization of this problem. Let $S_{d}$ be a $d$-dimensional simplex, $S_{d} \subset \mathbb{R}^{d}$, and let $H$ be an affine hyperplane of $\mathbb{R}^{d}$ given so that the distance between $H$ and any vertex of $S_{d}$ is the same constant. We say that such a hyperplane $H$ is a medial hyperplane of $S_{d}$. Let us define the medial section of $S_{d}$ with respect to $H$ as the $(d-1)$-dimensional convex body $S_{d} \cap H$. Then the analogous question is whether there exists a "simple" formula for the $(d-1)$-dimensional volume of the medial section $S_{d} \cap H$ in terms of the lengths of the edges of $S_{d}$.

\footnotetext{
* Current address: Department of Geometry, Eötvös University, P.O. Box 120, Budapest, Hungary. talata@cs.elte.hu.
} 
The answer to this question is affirmative and it turns out that the square of the volume of a medial section is a polynomial of the squares of the edge lengths of the simplex $S_{d}$. For the sake of nicer formularization, instead of the volume we express the square of the volume in the following.

Theorem 1. Let $S_{d}$ be a simplex in $\mathbb{R}^{d}$ with vertices $v_{1}, \ldots, v_{d+1}$, and let $H$ be a medial hyperplane of $S_{d}$ separating $\left\{v_{1}, \ldots, v_{m}\right\}$ from $\left\{v_{m+1}, \ldots, v_{d+1}\right\}$. Then

$$
\operatorname{Vol}_{d-1}^{2}\left(S_{d} \cap H\right)=\frac{1}{(-8)^{d-1}((m-1) !)^{2}((d-m) !)^{2}} \operatorname{det} M_{d+3}^{(m)},
$$

where $M_{d+3}^{(m)}$ is a symmetric $(d+3) \times(d+3)$ matrix with block form

$$
M_{d+3}^{(m)}=\left(\begin{array}{cc}
O_{2} & I_{d+1}^{(m)} \\
I_{d+1}^{(m) T} & A_{d+1}
\end{array}\right)
$$

in which $\mathrm{O}_{2}$ is a $2 \times 2$ zero-matrix, $I_{d+1}^{(m)}$ is a $2 \times(d+1)$ matrix of form

$$
I_{d+1}^{(m)}=\left(\begin{array}{cccccc}
1 & \cdots & 1 & 0 & \cdots & 0 \\
0 & \cdots & 0 & \underbrace{1}_{m} & \cdots & 1
\end{array}\right)
$$

with $m$ consecutive 1 's and $(d+1-m)$ consecutive 0 's in the first row, and $m$ consecutive 0 's and $(d+1-m)$ consecutive 1 's in the second row, and $A_{d+1}=\left\{a_{i, j}^{2}\right\}$ is a $(d+1) \times$ $(d+1)$ matrix with entries $a_{i, j}^{2}$, where $a_{i, j}$ measures the distance between $v_{i}$ and $v_{j}$.

Note that for $d=3$ and $m=2$ formula (1) of Theorem 1 gives the square of the area of a medial parallelogram $P$ of the tetrahedron $S_{3}$. However, one needs to write out the determinant in (1) and to do some algebraic simplification to get

$$
\operatorname{Area}^{2}(P)=\frac{1}{64}\left(a_{1,2}^{2} a_{3,4}^{2}-\left(a_{1,4}^{2}-a_{4,2}^{2}+a_{2,3}^{2}-a_{3,1}^{2}\right)^{2}\right),
$$

which is the formula of Yetter [5].

Recall the well-known formula

$$
\operatorname{Vol}^{2}\left(S_{d}\right)=\frac{(-1)^{d+1}}{2^{d}(d !)^{2}} \operatorname{det} M_{d+2},
$$

where $M_{d+2}$ is the $(d+2) \times(d+2)$ matrix with block form

$$
M_{d+2}=\left(\begin{array}{cc}
0 & 1_{d+1} \\
1_{d+1}^{T} & A_{d+1}
\end{array}\right)
$$

(see [1] and [4]), in which $1_{d+1}$ is the row matrix of length $d+1$ with all entries equal to 1 , and $A_{d+1}$ is the same matrix as in Theorem 1. The determinant in (3) is the socalled Cayley-Menger determinant. The volume formula (3) has various applications in geometry, see [2] and [3] for some examples.

Since (1) and (3) are similar formulas for squares of certain volumes, and both contain analogous determinant factors, one may suspect that there is a common generalization 
of these formulas that still expresses the square of the $l$-volume of the intersection of $S_{d}$ and an analogously chosen "medial" $l$-dimensional affine subspace of $\mathbb{R}^{d}$, for any $0 \leq l \leq d$. In the following we describe such a generalization which includes both (1) and (3) as special cases.

Let $\mathcal{P}=\left\{V_{1}, V_{2}, \ldots, V_{k}\right\}$ be a $k$-partition of the vertex set $\operatorname{vert}\left(S_{d}\right)$, for which

$$
V_{i}=\left\{v_{n_{i-1}+1}, v_{n_{i-1}+2}, \ldots, v_{n_{i}}\right\}
$$

holds for every $1 \leq i \leq k$, with $0=n_{0}<n_{1}<\cdots<n_{k}=d+1$. Let $m_{i}=n_{i}-n_{i-1}$. Clearly, each $V_{i}$ has cardinality $m_{i}$. This means that

$$
\mathcal{P}=\left\{\left\{v_{1}, \ldots, v_{m_{1}}\right\},\left\{v_{m_{1}+1}, \ldots, v_{m_{1}+m_{2}}\right\}, \ldots,\left\{v_{d+2-m_{k}}, \ldots, v_{d+1}\right\}\right\} .
$$

That is, $\mathcal{P}$ is a collection of $k$ disjoint nonempty subsets of $\operatorname{vert}(P)$ each containing consecutively labeled vertices. Let $P_{i}=\operatorname{aff}\left(V_{i}\right)$ for every $1 \leq i \leq k$, where aff() stands for the affine hull. Let $H^{\prime}=\frac{1}{k} \sum_{i=1}^{k} P_{i}$. In other words, $H^{\prime}$ is the Minkowski sum (or set theoretical sum) of the $P_{i}$ 's rescaled by a homothety with factor $1 / k$ (for an exact definition of Minkowski sums, see Section 2.1). Since each $P_{i}$ is an affine subspace of $\mathbb{R}^{d}$ of dimension $m_{i}-1$, therefore $H^{\prime}$ is an affine subspace of dimension $\sum_{i=1}^{k}\left(m_{i}-1\right)=d+1-k$. The following holds.

Theorem 2. Let $\mathcal{P}$ be a $k$-partition of the vertices of a d-dimensional simplex $S_{d}$ as described previously $(1 \leq k \leq d+1)$. Using the notation above, we have

$$
\operatorname{Vol}_{d+1-k}^{2}\left(S_{d} \cap H^{\prime}\right)=\frac{(-1)^{d+1}}{\left(2 k^{2}\right)^{d+1-k} \prod_{i=1}^{k}\left(\left(m_{i}-1\right) !\right)^{2}} \operatorname{det} M_{\mathcal{P}},
$$

where $M_{\mathcal{P}}$ is a symmetric $(d+k+1) \times(d+k+1)$ matrix with blockform

$$
M_{\mathcal{P}}=\left(\begin{array}{cc}
O_{k} & I_{\mathcal{P}} \\
I_{\mathcal{P}}^{T} & A_{d+1}
\end{array}\right)
$$

in which $O_{k}$ is a $k \times k$ zero-matrix, $I_{\mathcal{P}}=\left\{g_{i, j}\right\}$ is a $k \times(d+1)$ zero-one matrix with entries

$$
g_{i, j}= \begin{cases}1, & \text { if } n_{i-1}+1 \leq j \leq n_{i}, \\ 0, & \text { otherwise, }\end{cases}
$$

and $A_{d+1}$ is the same $(d+1) \times(d+1)$ matrix as described in Theorem 1 .

Note that in Theorem 2 the entries of $I_{\mathcal{P}}$ are defined so that we have $g_{i, j}=1$ if and only if $v_{j} \in V_{i}$. Therefore $I_{\mathcal{P}}$ is the characteristic matrix of the $k$-partition $\mathcal{P}$ of $\operatorname{vert}\left(S_{d}\right)$. It is also clear that $I_{\mathcal{P}}$ is a generalization of the matrix $I_{d+1}^{(m)}$ of Theorem 1. For example, for $k=3$ we have

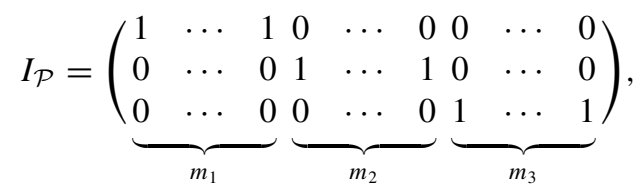

which is analogous to (2). 
Observe that $H^{\prime}$ of Theorem 2 is a generalization of $H$ of Theorem 1 since the latter can be written as

$$
H=\frac{1}{2}\left(\operatorname{aff}\left(v_{1}, v_{2}, \ldots, v_{m}\right)+\operatorname{aff}\left(v_{m+1}, \ldots, v_{d+1}\right)\right) .
$$

Therefore one may call $H^{\prime}$ a medial $(d+1-k)$-dimensional affine subspace of $S_{d}$, and $S_{d} \cap H^{\prime}$ may be called a medial $(d+1-k)$-section of $S_{d}$.

We remark that Theorem 1 is a special case of Theorem 2 with $k=2$ and $m_{1}=m$, while (3) is a special case of Theorem 2 with $k=1$. However, we prove only Theorem 1 in detail, and for the proof of Theorem 2 just a sketch is given, since the latter goes the same way as the proof of Theorem 1 . Only the more extensive notation needed there would make a detailed proof of Theorem 2 more lengthy and complicated.

\section{Proofs}

\subsection{Notation}

If $K$ is an $l$-dimensional convex subset in $\mathbb{R}^{d}$ for some $l, 0 \leq l \leq d$, then we denote its $l$-dimensional volume by $\operatorname{Vol}_{l}(K)$. For $l=d$ we use the notation $\operatorname{Vol}(K)$.

If $U, V \subseteq \mathbb{R}^{d}$, then let $U+V=\left\{u+v \in \mathbb{R}^{d} \mid u \in U, v \in V\right\}$, that is, $U+V$ is the Minkowski sum of $U$ and $V$. For $v \in \mathbb{R}^{d}$ we simply write $U+v$ instead of $U+\{v\}$. For $U \subseteq \mathbb{R}^{d}$ and $\alpha \in \mathbb{R}$, let $\alpha U$ be the set $\{\alpha u \mid u \in U\}$. If $a, b \in \mathbb{R}^{d}$, then both $[a, b]$ and $a b$ denote the segment with endpoints $a$ and $b$. We denote by $o$ the orgin of $\mathbb{R}^{d}$.

Throughout the paper conv( ) and aff( ) stand for the convex hull and the affine hull, respectively. For a convex polyhedron $P$ we use the notation vert $(P)$ for its vertex set. For $u \in \mathbb{R}^{d}$ we denote its Euclidean norm by $\|u\|$. If $U$ is a linear subspace of $\mathbb{R}^{d}$, then $U^{\perp}$ is the orthogonal complement of $U$ in $\mathbb{R}^{d}$.

We denote by $1_{l},-1_{l}$ and $0_{l}$ the row matrices of length $l$ with all entries equal to 1 , -1 and 0 , respectively. If $M$ is a matrix, then we use the notation $M^{\mathrm{T}}$ for the matrix transposed to $M$.

\subsection{Proof of Theorem 1}

Throughout this part we assume that the conditions of Theorem 1 hold. Our goal is to prove (1). Let $C_{1}=\operatorname{conv}\left(v_{1}, \ldots, v_{m}\right)$ and $C_{2}=\operatorname{conv}\left(v_{m+1}, \ldots, v_{d+1}\right)$. Then $C_{1}$ is an $(m-1)$-dimensional simplex and $C_{2}$ is a $(d-m)$-dimensional simplex. Let $C_{1}^{\prime}=C_{1}-v_{m}$ and $C_{2}^{\prime}=C_{2}-v_{d+1}$. Since $S_{d}=\operatorname{conv}\left(C_{1} \cup C_{2}\right)$ and $H=\frac{1}{2}\left(\operatorname{aff}\left(C_{1}\right)+\operatorname{aff}\left(C_{2}\right)\right)$, therefore $S_{d} \cap H=\frac{1}{2}\left(C_{1}+C_{2}\right)$. This means that $S_{d} \cap H$ is a translate of $\frac{1}{2}\left(C_{1}^{\prime}+C_{2}^{\prime}\right)$.

On the other hand, $D_{1}=\sum_{i=1}^{m-1}\left[o, v_{i}-v_{m}\right]$ and $D_{2}=\sum_{i=m+1}^{d}\left[o, v_{i}-v_{d+1}\right]$ are paralleletopes, and their Minkowski sum $D=D_{1}+D_{2}$ is also a paralleletope. Let

$$
v_{i}^{\prime}=\left\{\begin{array}{lll}
v_{i}-v_{m}, & \text { if } \quad 1 \leq i \leq m-1, \\
v_{i+1}-v_{d+1}, & \text { if } \quad m \leq i \leq d-1
\end{array}\right.
$$


Let

$$
C^{\prime}=\operatorname{conv}\left(o,\left\{v_{i}^{\prime} \mid 1 \leq i \leq d-1\right\}\right) .
$$

Then $C^{\prime}$ is a $(d-1)$-dimensional simplex.

We have

$$
\begin{aligned}
\operatorname{Vol}_{m-1}\left(D_{1}\right) & =(m-1) ! \operatorname{Vol}_{m-1}\left(C_{1}^{\prime}\right), \\
\operatorname{Vol}_{d-m}\left(D_{2}\right) & =(d-m) ! \operatorname{Vol}_{d-m}\left(C_{2}^{\prime}\right), \\
\operatorname{Vol}_{d-1}(D) & =(d-1) ! \operatorname{Vol}_{d-1}\left(C^{\prime}\right),
\end{aligned}
$$

and

$$
\begin{aligned}
\operatorname{Vol}_{d-1}(D) & =\operatorname{Vol}_{m-1}\left(D_{1}\right) \alpha \operatorname{Vol}_{d-m}\left(D_{2}\right), \\
\operatorname{Vol}_{d-1}\left(C_{1}^{\prime}+C_{2}^{\prime}\right) & =\operatorname{Vol}_{m-1}\left(C_{1}^{\prime}\right) \alpha \operatorname{Vol}_{d-m}\left(C_{2}^{\prime}\right),
\end{aligned}
$$

for some constant $\alpha>0$, since the affine hulls of $D_{1}$ and $D_{2}$ are the same as the affine hulls of $C_{1}^{\prime}$ and $C_{2}^{\prime}$, respectively, so $\alpha \operatorname{Vol}_{d-m}\left(D_{2}\right)$ is the $(d-m)$-volume of the parallel projection of $D_{2}$ to aff $\left(D_{1}\right)^{\perp}$ and $\alpha \operatorname{Vol}_{d-m}\left(C_{2}^{\prime}\right)$ is the $(d-m)$-volume of the parallel projection of $C_{2}^{\prime}$ to $\operatorname{aff}\left(C_{1}\right)^{\perp}$, if $\alpha$ is chosen appropriately.

From these equalities follows

$$
\begin{aligned}
\operatorname{Vol}_{d-1}\left(S_{d} \cap H\right)=\frac{1}{2^{d-1}} \operatorname{Vol}_{d-1}\left(C_{1}^{\prime}+C_{2}^{\prime}\right) & =\frac{\operatorname{Vol}_{m-1}\left(C_{1}^{\prime}\right) \operatorname{Vol}_{d-m}\left(C_{2}^{\prime}\right) \operatorname{Vol}_{d-1}(D)}{2^{d-1} \operatorname{Vol}_{m-1}\left(D_{1}\right) \operatorname{Vol}_{d-m}\left(D_{2}\right)} \\
& =\frac{(d-1) ! \operatorname{Vol}_{d-1}\left(C^{\prime}\right)}{2^{d-1}(m-1) !(d-m) !} .
\end{aligned}
$$

Applying (3) for the factor $\operatorname{Vol}_{d-1}\left(C^{\prime}\right)$ in the previous expression we get

$$
\mathrm{Vol}_{d-1}^{2}\left(S_{d} \cap H\right)=\frac{(-1)^{d} \operatorname{det} N_{d+1}}{8^{d-1}((m-1) !)^{2}((d-m) !)^{2}},
$$

where $N_{d+1}$ is a $(d+1) \times(d+1)$ matrix with block form

$$
N_{d+1}=\left(\begin{array}{cc}
0 & 1_{d} \\
1_{d}^{\mathrm{T}} & B_{d}
\end{array}\right)
$$

with $B_{d}=\left\{b_{i, j}^{2}\right\}_{i, j=1}^{d}$, where $b_{i, j}$ is the distance between $v_{i-1}^{\prime}$ and $v_{j-1}^{\prime}$, for $1 \leq i, j \leq d$ (with the notation $v_{0}^{\prime}=o$ ). It is clear that to complete the proof of Theorem 1 we only need to show that

$$
\operatorname{det} M_{d+3}^{(m)}=-\operatorname{det} N_{d+1} .
$$

To prove this, we need the following.

Lemma 3. Let $T$ be a tetrahedron in $\mathbb{R}^{3}$ with vertices $p_{1}, p_{2}, p_{3}$ and $p_{4}$. Let $h$ be the length of the segment connecting the midpoints of the two opposite edges $p_{1} p_{2}$ and $p_{3} p_{4}$ of $T$. If $s_{i, j}$ denotes the distance between the vertices $p_{i}$ and $p_{j}$ of $T, 1 \leq i, j \leq 4$, then we have

$$
h^{2}=\frac{1}{4}\left(s_{1,3}^{3}+s_{3,2}^{2}+s_{2,4}^{2}+s_{4,1}^{2}-s_{1,2}^{2}-s_{3,4}^{2}\right) .
$$


Proof. Let $c$ be the baricenter of $T$, that is, $c=\frac{1}{4}\left(p_{1}+p_{2}+p_{3}+p_{4}\right)$. Let $T^{\prime}$ be the centrally reflected image of $T$ with center $c$. Denote by $p_{i}^{\prime}$ that vertex of $T^{\prime}$ which is the centrally reflected image of $p_{i}$, for any $1 \leq i \leq 4$. Since $c$ is clearly the midpoint of any segment connecting the midpoints of two opposite edges of $T^{\prime}$, therefore for any two opposite edges $p_{i} p_{j}$ and $p_{k} p_{l}$ of $T$ it holds that the segment $p_{i}^{\prime} p_{j}^{\prime}$ intersects $p_{k} p_{l}$ in a point which is the midpoint of both segments, and consequently $p_{i}^{\prime} p_{k} p_{j}^{\prime} p_{l}$ is a parallelogram. This means that

$$
Q=\operatorname{conv}\left(p_{1}, p_{2}, p_{3}, p_{4}, p_{1}^{\prime}, p_{2}^{\prime}, p_{3}^{\prime}, p_{4}^{\prime}\right)
$$

is a parallelepiped, and $\operatorname{vert}(Q)=\operatorname{vert}(T) \cup \operatorname{vert}\left(T^{\prime}\right)$. Let $e_{2}, e_{3}$ and $e_{4}$ be the lengths of the edges $p_{1} p_{2}^{\prime}, p_{1} p_{3}^{\prime}$ and $p_{1} p_{4}^{\prime}$ of $Q$, respectively. We recall the well-known fact that the sum of the squares of the four sides af any parallelogram equals the sum of the squares of the two diagonals of that parallelogram. Using this fact for the three faces $p_{1} p_{2}^{\prime} p_{4} p_{3}^{\prime}$, $p_{1} p_{2}^{\prime} p_{3} p_{4}^{\prime}$ and $p_{1} p_{3}^{\prime} p_{2} p_{4}^{\prime}$ of $Q$ we get

$$
\begin{aligned}
& 2\left(e_{2}^{2}+e_{3}^{2}\right)=s_{3,2}^{2}+s_{4,4}^{2}, \\
& 2\left(e_{2}^{2}+e_{4}^{2}\right)=s_{1,3}^{2}+s_{2,4}^{2}, \\
& 2\left(e_{3}^{2}+e_{4}^{2}\right)=s_{1,2}^{2}+s_{3,4}^{2} .
\end{aligned}
$$

Summing up the first two equations, and subtracting the third one from the sum, we obtain

$$
4 e_{2}^{2}=s_{1,3}^{2}+s_{3,2}^{2}+s_{2,4}^{2}+s_{4,1}^{2}-s_{1,2}^{2}-s_{3,4}^{2} .
$$

Finally, observe that $h=e_{2}$. Thus, after dividing both sides of (8) by 4 , and replacing $e_{2}$ with $h$, we get (7).

We now continue the proof of Theorem 1. By (5) we have the following block form for $B_{d}$ :

$$
B_{d}=\left(\begin{array}{ccc}
0 & w_{1} & w_{2} \\
w_{1}^{\mathrm{T}} & X & Y \\
w_{2}^{\mathrm{T}} & Y^{\mathrm{T}} & Z
\end{array}\right),
$$

where $w_{1}$ and $w_{2}$ are row matrices of lengths $m-1$ and $d-m$, respectively, of form

$$
\begin{aligned}
& w_{1}=\left(a_{1, m}^{2}, a_{2, m}^{2}, \ldots, a_{m-1, m}^{2}\right), \\
& w_{2}=\left(a_{m+1, d+1}^{2}, a_{m+2, d+1}^{2}, \ldots, a_{d, d+1}^{2}\right),
\end{aligned}
$$

and $X=\left\{x_{i, j}\right\}, Y=\left\{y_{i, j}\right\}$ and $Z=\left\{z_{i, j}\right\}$ are matrices of dimensions $(m-1) \times(m-1)$, $(m-1) \times(d-m)$ and $(d-m) \times(d-m)$, respectively, with entries

$$
\begin{array}{ll}
x_{i, j}=a_{i, j}^{2} & \text { for } \quad 1 \leq i, j \leq m-1, \\
y_{i, j}=b_{i+1, m+j}^{2} & \text { for } \quad 1 \leq i \leq m-1 \quad \text { and } \quad 1 \leq j \leq d-m, \\
z_{i, j}=a_{m+i, m+j}^{2} & \text { for } \quad 1 \leq i, j \leq d-m .
\end{array}
$$


Since

$$
y_{i, j}=b_{i+1, m+j}^{2}=4\left\|\frac{v_{i}+v_{d+1}}{2}-\frac{v_{m+j}+v_{m}}{2}\right\|^{2},
$$

thus by Lemma 3 we have

$$
y_{i, j}=a_{i, m+j}^{2}+a_{m+j, d+1}^{2}+a_{m, d+1}^{2}+a_{i, m}^{2}-a_{i, d+1}^{2}-a_{m, m+j}^{2} .
$$

It is evident that $\operatorname{det} N_{d+1}=\operatorname{det} \widehat{M}$, where $\widehat{M}$ is a $(d+3) \times(d+3)$ matrix with block form

$$
\widehat{M}=\left(\begin{array}{cccccc}
1 & 0 & 0 & 0 & 0_{m-1} & 0_{d-m} \\
0 & 1 & 1 & 0 & 0_{m-1} & w_{2} \\
1 & 0 & 0 & 1 & 1_{m-1} & 1_{d-m} \\
0 & 0 & 1 & 0 & w_{1} & w_{2} \\
0_{m-1}^{\mathrm{T}} & 0_{m-1}^{\mathrm{T}} & 1_{m-1}^{\mathrm{T}} & w_{1}^{\mathrm{T}} & X & Y \\
w_{2}^{\mathrm{T}} & 0_{d-m}^{\mathrm{T}} & 1_{d-m}^{\mathrm{T}} & w_{2}^{\mathrm{T}} & Y^{\mathrm{T}} & Z
\end{array}\right)
$$

We will use row and column operations to alter $\widehat{M}$ until we get $M_{d+3}^{(m)}$. Namely, we will multiply some rows and columns by -1 , we will add some multiples of rows to other rows and some multiples of columns to other columns, and furthermore, we will reposition some rows and columns. Clearly, the determinant of such a newly obtained matrix will be $\pm \operatorname{det} \widehat{M}$, and the sign will depend on the parity of the total change of indices of the rows and columns repositioned, and on the number of rows and columns multiplied by -1 .

In the following we give a list of 16 row and column operations which transform $\widehat{M}$ into $M_{d+3}^{(m)}$. At each step, denote by $R_{i}$ and $C_{i}$ the $i$ th row and column of the actual matrix, respectively. By the actual matrix we mean that matrix which is obtained from $\widehat{M}$ as the result of those steps already made. Note that this means that in different steps $R_{i}$ and $C_{i}$ may stand for different rows and columns for some $i$. We use arrow notation, e.g. $C_{i} \rightarrow C_{i}-C_{1}$ in Step 1 means that a new $i$ th column is obtained after applying this step by subtracting the 1 st column from the $i$ th column of the actual matrix. Here is the list of the row and column operations:

Step 1. $C_{i} \rightarrow C_{i}-C_{1}$ for $4 \leq i \leq m+3$.

Step 2. $R_{i} \rightarrow R_{i}-R_{2}$ for $4 \leq i \leq m+3$.

Step 3. $C_{i} \rightarrow C_{i}-C_{4}$ for $m+4 \leq i \leq d+3$.

Step 4. $R_{i} \rightarrow R_{i}-R_{4}$ for $m+4 \leq i \leq d+3$.

Step 5. $C_{4}$ is shifted to between $C_{m+3}$ and $C_{m+4}$.

Step 6. $R_{4}$ is shifted to between $R_{m+3}$ and $R_{m+4}$.

Step 7. $C_{1}$ is shifted to be the last column.

Step 8. $R_{2}$ is shifted to be the last row.

Step 9. $C_{1} \rightarrow-C_{1}+C_{2}$.

Step 10. $R_{1} \rightarrow-R_{1}+R_{2}$.

Step 11. $C_{i} \rightarrow a_{m, i-2}^{2} C_{1}+C_{i}$ for $m+3 \leq i \leq d+2$.

Step 12. $R_{i} \rightarrow a_{m, i-2}^{2} R_{1}+R_{i}$ for $m+3 \leq i \leq d+2$.

Step 13. $C_{i} \rightarrow\left(a_{i-2, d+1}^{2}-a_{m, d+1}^{2}\right) C_{2}+C_{i}$ for $3 \leq i \leq m+1$. 
Step 14. $R_{i} \rightarrow\left(a_{i-2, d+1}^{2}-a_{m, d+1}^{2}\right) R_{2}+R_{i}$ for $3 \leq i \leq m+1$.

Step 15. $C_{d+3} \rightarrow a_{m, d+1}^{2} C_{1}+C_{d+3}$.

Step 16. $R_{d+3} \rightarrow a_{m, d+1}^{2} R_{1}+R_{d+3}$.

Now we describe how the matrix $\widehat{M}$ is affected by the row and column operations of the steps above.

After Steps 1 and 2 we get the following matrix $\widehat{M}_{1}$ with block form

$$
\widehat{M}_{1}=\left(\begin{array}{cccccc}
1 & 0 & 0 & -1 & -1_{m-1} & 0_{d-m} \\
0 & 1 & 1 & 0 & 0_{m-1} & w_{2} \\
1 & 0 & 0 & 0 & 0_{m-1} & 1_{d-m} \\
0 & -1 & 0 & 0 & w_{1} & 0_{d-m} \\
0_{m-1}^{\mathrm{T}} & -1_{m-1}^{\mathrm{T}} & 0_{m-1}^{\mathrm{T}} & w_{1}^{\mathrm{T}} & X & Y_{1} \\
w_{2}^{\mathrm{T}} & 0_{d-m}^{\mathrm{T}} & 1_{d-m}^{\mathrm{T}} & 0_{d-m}^{\mathrm{T}} & Y_{1}^{\mathrm{T}} & Z
\end{array}\right)
$$

in which $Y_{1}$ is a submatrix with entries defined similarly to (9) except that the second term of the right side of (9) is dropped out.

After Steps 3 and 4 we get

$$
\widehat{M}_{2}=\left(\begin{array}{cccccc}
1 & 0 & 0 & -1 & -1_{m-1} & 1_{d-m} \\
0 & 1 & 1 & 0 & 0_{m-1} & w_{2} \\
1 & 0 & 0 & 0 & 0_{m-1} & 1_{d-m} \\
0 & -1 & 0 & 0 & w_{1} & 0_{d-m} \\
0_{m-1}^{\mathrm{T}} & -1_{m-1}^{\mathrm{T}} & 0_{m-1}^{\mathrm{T}} & w_{1}^{\mathrm{T}} & X & Y_{2} \\
w_{2}^{\mathrm{T}} & 1_{d-m}^{\mathrm{T}} & 1_{d-m}^{\mathrm{T}} & 0_{d-m}^{\mathrm{T}} & Y_{2}^{\mathrm{T}} & Z
\end{array}\right)
$$

in which $Y_{2}$ is a submatrix with entries defined similarly to (9) except that the second and the fourth terms of the right side of (9) are dropped out.

After Steps 5 through 8 we get

$$
\widehat{M}_{3}=\left(\begin{array}{cccccc}
0 & 0 & -1_{m-1} & -1 & 1_{d-m} & 1 \\
0 & 0 & 0_{m-1} & 0 & 1_{d-m} & 1 \\
-1_{m-1}^{\mathrm{T}} & 0_{m-1}^{\mathrm{T}} & X & w_{1}^{\mathrm{T}} & Y_{2} & 0_{m-1}^{\mathrm{T}} \\
-1 & 0 & w_{1} & 0 & 0_{d-m} & 0 \\
1_{d-m}^{\mathrm{T}} & 1_{d-m}^{\mathrm{T}} & Y_{2}^{\mathrm{T}} & 0_{d-m}^{\mathrm{T}} & Z & w_{2}^{\mathrm{T}} \\
1 & 1 & 0_{m-1} & 0 & w_{2} & 0
\end{array}\right)
$$

in which some rows and columns are repositioned in such a way that the total change of indices of repositioned rows and columns is $(m-1)+(m-1)+(d+2)+(d+1)=$ $2 m+2 d+1$, which is an odd number. Therefore $\operatorname{det} \widehat{M}_{3}=-\operatorname{det} \widehat{M}_{2}$. 
After Steps 9 and 10 we get

$$
\widehat{M}_{4}=\left(\begin{array}{cccccc}
0 & 0 & 1_{m-1} & 1 & 0_{d-m} & 0 \\
0 & 0 & 0_{m-1} & 0 & 1_{d-m} & 1 \\
1_{m-1}^{\mathrm{T}} & 0_{m-1}^{\mathrm{T}} & X & w_{1}^{\mathrm{T}} & Y_{2} & 0_{m-1}^{\mathrm{T}} \\
1 & 0 & w_{1} & 0 & 0_{d-m} & 0 \\
0_{d-m}^{\mathrm{T}} & 1_{d-m}^{\mathrm{T}} & Y_{2}^{\mathrm{T}} & 0_{d-m}^{\mathrm{T}} & Z & w_{2}^{\mathrm{T}} \\
0 & 1 & 0_{m-1} & 0 & w_{2} & 0
\end{array}\right) .
$$

By Steps 11 through 14, $Y_{2}$ is modified so that after Step 14 it has the desired form $Y_{2}=\left\{a_{i, m+j}^{2}\right\}$. However, in the last column and in the last row of the actual matrix some undesired terms remain, which are canceled out by Steps 15 and 16. After these last steps we get the matrix $M_{d+3}^{(m)}$. Since the sign of the determinant did not change except Steps 5 through 10 , and obviously we have $\operatorname{det} \widehat{M}_{3}=\operatorname{det} \widehat{M}_{4}$, therefore it holds that

$$
\operatorname{det} N_{d+1}=\operatorname{det} \widehat{M}=\operatorname{det} \widehat{M}_{2}=-\operatorname{det} \widehat{M}_{3}=-\operatorname{det} \widehat{M}_{4}=-\operatorname{det} M_{d+3}^{(m)} .
$$

This completes the proof of Theorem 1.

\subsection{Proof of Theorem 2}

We only give an outline of the proof since the more general settings of Theorem 2 would make a detailed proof lengthy and complicated. Apart from this, a proof analogous to the proof of Theorem 1 can be given. We now indicate the main steps of such a proof, pointing out how they are analogous to the corresponding ones of Theorem 1.

For the given $k$-partition $\mathcal{P}=\left\{V_{1}, V_{2}, \ldots, V_{k}\right\}$, one can define $C_{j}=\operatorname{conv}\left(V_{j}\right)$ for $1 \leq j \leq k$, and $C_{j}^{\prime}=C_{j}-v_{n_{j}}$. Let $C^{\prime}=\operatorname{conv}\left(\bigcup_{j=1}^{k} \operatorname{vert}\left(C_{j}^{\prime}\right)\right)$. Then $C^{\prime}$ is a $(d+1-k)$ dimensional simplex in $\mathbb{R}^{d}$. Analogously to the proof of Theorem 1, using corresponding paralleletopes to each $C_{j}^{\prime}$ and to $C^{\prime}, \operatorname{Vol}_{d+1-k}\left(S_{d} \cap H^{\prime}\right)$ can be expressed as a multiple of $\mathrm{Vol}_{d+1-k}\left(C^{\prime}\right)$ with a coefficient depending only on $d, k$ and $m_{i}$ 's $(1 \leq i \leq k)$. Applying (3) for $\operatorname{Vol}_{d+1-k}\left(C^{\prime}\right)$, a formula analogous to (6) can be found, by which the proof is reduced to the justification of $\operatorname{det} M_{\mathcal{P}}=(-1)^{k-1} \operatorname{det} N_{d+3-k}$, where $N_{d+3-k}$ is a certain $(d+3-k) \times(d+3-k)$ matrix, having block form

$$
N_{d+3-k}=\left(\begin{array}{ccc}
0 & 1 & 1_{d+1-k} \\
1 & 0 & z \\
1_{d+1-k}^{\mathrm{T}} & z^{\mathrm{T}} & U
\end{array}\right),
$$

where $z$ is a row matrix of block form $z=\left(w_{1}, w_{2}, \ldots, w_{k}\right)$, with the notation

$$
w_{i}=\left(a_{n_{i}-m_{i}+1, n_{i}}^{2}, a_{n_{i}-m_{i}+2, n_{i}}^{2}, \ldots, a_{n_{i}-1, n_{i}}^{2}\right), \quad 1 \leq i \leq k .
$$

Analogously to (10), $N_{d+3-k}$ can be expanded with $2 k-2$ new rows and columns so that its determinant remains unchanged. The expanded matrix can be written in the following 
block form:

$$
\hat{M}_{\mathcal{P}}=\left(\begin{array}{ccc}
I_{k-1} & O_{k-1} & O_{k-1, d+3-k} \\
O_{k-1} & I_{k-1} & V \\
V^{\mathrm{T}} & O_{d+3-k, k-1} & N_{d+3-k}
\end{array}\right)
$$

where $V$ is the $(k-1) \times(d+3-k)$-dimensional matrix whose $i$ th row has block form

$$
\left(0_{n_{i}-i+2}, w_{i+1}, 0_{d+4+i-k-n_{i+1}}\right), \quad 1 \leq i \leq k-1 .
$$

We also used the notation $I_{r}$ and $O_{r, s}$ for unit square matrices and zero matrices, respectively, whose dimensions are shown in their indices. Next, the entries of the submatrix $U$ of $N_{d+3-k}$ can be calculated by Lemma 3, having forms similar to (9). Finally, one can use row and column operations to get $M_{\mathcal{P}}$ from $\hat{M}_{\mathcal{P}}$. By counting the parity of the total change of indices during the row and column operations one can establish $\operatorname{det} N_{d+3-k}=(-1)^{k-1} \operatorname{det} M_{\mathcal{P}}$.

\section{Concluding Remarks}

It is worth mentioning that a medial section $S_{d} \cap H^{\prime}$ of Theorem 2 may belong to classes of objects of really different shapes, depending on the choice of $d, k$ and $m_{i}$ 's $(1 \leq i \leq k)$. As extremal cases, for $k=1$ and $k=d+1$ we get $S_{d}$ itself and its baricenter as a single point, respectively. Also, certain segments, triangles and parallelograms can be obtained as medial sections. In general, every medial section can be obtained as the Minkowski sum of certain simplices whose vertices form a partition of the vertices of some $d$ dimensional simplex. We mention two nonplanar examples: for $d=4, k=2, m_{1}=3$ and $m_{2}=2$, the medial section is a three-dimensional (possibly skew) cylinder with a triangular base, while for $d=5, k=2$ and $m_{1}=m_{2}=3$ we get a four-dimensional convex polytope with six facets of (possibly skew) cylinders with triangular bases.

The question arises whether there is a volume formula for other, not necessarily medial sections of $S_{d}$, obtained as the intersection of $S_{d}$ and some affine subspace of $\mathbb{R}^{d}$. The answer is positive if the affine subspace is parallel to a medial affine subspace of $S_{d}$ and it intersects the interior of $S_{d}$. In fact, then the affine subspace can be written in a form $\widehat{H}=\sum_{i=1}^{k} \alpha_{i}$ aff $\left(V_{i}\right)$ for some positive coefficients $\alpha_{i}, 1 \leq i \leq k$, with $\sum_{i=1}^{k} \alpha_{i}=1$. Thus

$$
\operatorname{Vol}_{d+1-k}\left(S_{d} \cap \widehat{H}\right)=\left(\prod_{i=1}^{k} \alpha_{i}^{m_{i}-1}\right) \operatorname{Vol}_{d+1-k}\left(S_{d} \cap H^{\prime}\right)
$$

and the explicit formula can be found by substituting (4) into the right side. However, it is not clear at all if there is a formula of similar kind for the volumes of intersections of $S_{d}$ and generally positioned affine subspaces. We pose this question in the following.

Problem. Let $S_{d}$ be a $d$-dimensional simplex vith vertices $\left\{v_{1}, v_{2}, \ldots, v_{d+1}\right\}$ in $\mathbb{R}^{d}$, and let $\widetilde{H}$ be an arbitrary $l$-dimensional affine subspace of $\mathbb{R}^{d}$ for some $l, 1 \leq l \leq d-1$, 
given so that $\widetilde{H}$ intersects the interior of $S_{d}$. Let $\widetilde{H}^{*}$ be the $l$-dimensional affine subspace of $\mathbb{R}^{d+1}$ defined as

$$
\widetilde{H}^{*}=\left\{\left(\alpha_{1}, \alpha_{2}, \ldots, \alpha_{d+1}\right) \in \mathbb{R}^{d+1} \mid \sum_{i=1}^{d+1} \alpha_{i} v_{i} \in \widetilde{H}, \sum_{i=1}^{d+1} \alpha_{i}=1\right\},
$$

which encodes the position of $\widetilde{H}$ relative to $S_{d}$ in an affine invariant way.

Is there an algebraic formula expressing $\operatorname{Vol}_{l}\left(S_{d} \cap \widetilde{H}\right)$ in terms of the lengths of the edges of $S_{d}$ and some parameters of $\widetilde{H}^{*}$ ?

\section{References}

1. L. M. Blumenthal, Theory and Applications of Distance Geometry, 2nd edn., Chelsea, New York, 1970.

2. R. Connelly, I. Sabitov and A. Walz, The Bellows conjecture, Beitr. Algebra Geom. 38 (1997), 1-10.

3. I. Kh. Sabitov, The volume as a metric invariant of polyhedra, Discrete Comput. Geom. 20 (1998), 405-428.

4. D. M. Y. Sommerville, An Introduction to the Geometry of n Dimensions, Dover, New York, 1958.

5. D. N. Yetter, The area of the medial parallelogram of a tetrahedron, Amer. Math. Monthly 106 (1999), 956-958.

Received September 13, 2000, and in revised form November 9, 2002. Online publication July 15, 2003. 